\title{
From classical to quantum Kagomé antiferromagnet in a magnetic field
}

\author{
D.C. Cabra ${ }^{1,2}$, M.D. Grynberg ${ }^{1}$, P.C.W. Holdsworth ${ }^{3}$ and P. Pujol ${ }^{3}$ \\ ${ }^{1}$ Departamento de Física, Universidad Nacional de la Plata, C.C. 67, (1900) La Plata, Argentina. \\ ${ }^{2}$ Facultad de Ingeniería, Universidad Nacional de Lomas de Zamora, Cno. de Cintura y Juan XXIII, \\ (1832) Lomas de Zamora, Argentina. \\ ${ }^{3}$ Laboratoire de Physique, ENS Lyon, 46 Allée d'Italie, 69364 Lyon Cédex 07, France.
}

\begin{abstract}
We study the magnetic properties of the Kagomé antiferromagnet going from the classical limit to the deep quantum regime of spin $1 / 2$ systems. In all the cases there are special values for the magnetization, $1 / 3$ in particular, in which a singular behavior is observed to occur in both the classical and quantum cases. We show clear evidence for a magnetization plateau for all $S$, in a wide range of $X X Z$ anisotropies and for the occurrence of quantum order by disorder effects.

PACS numbers: 75.10.Jm, 75.10.Nr, 75.60.Ej.
\end{abstract}

Spin systems in low dimensions have become an intense area of research in the last few years due to their possible relevance to high- $T_{c}$ cuprates but also in their own right due to their very special properties [1]. One of the issues that attracted attention, both from experimentalists and theorists, is the appearance of plateaux in the magnetization curves in both 1D 2, 4 and 2D [5 9 quantum spin systems. Another has been the study of strongly frustrated systems with highly degenerate classical ground state structure 10 giving disordered, spin liquid behavior at low temperature. The spin liquid behaviour can be removed, or partial removed by degeneracy lifting, through thermal, or quantum fluctuations; the phenomenon known as order by disorder (OBD) [11,12]. Examples are the so-called $J_{1}-J_{2}$ model on a square lattice 13. and the Kagomé Heisenberg antiferromagnet (AF), where thermal OBD leads to a nematic spin liquid state 14 16]. The situation is more complicated when one considers quantum spins defined on such lattices, and the role of quantum fluctuation in the selection of some particular ground states (GS's). In the particular case of the Kagomé lattice [17], state of the art numerical computations [18] seem to indicate that no nematic order is observed and that the GS is a rather complicated spin liquid like state, with a spin gap but with many low lying spinless states.

Recent studies of the Kagomé AF in the presence of a magnetic field have shown that the value of $1 / 3$ for the normalized magnetization is a special point bringing into play both OBD effects and the presence of a magnetic plateau, an effect also present for the triangular lattice [5,19]. In the classical case 20,21], spin wave analysis and Monte Carlo simulation show the order by disorder selection of a collinear spin-liquid state together with a magnetic anomaly and a pseudo magnetic plateau. In the quantum case, numerical evidence from the exact diagonalization of small $S=1 / 2$ clusters show the existence of a magnetic plateau [22. Such behavior has already been observed in the $J_{1}-J_{2}$ square lattice, where similar phenomena, based on a collinear order of the spins for particular values of the magnetization, occurs 20 .

A natural question one can pose is therefore: is there a connection between the existence of soft modes in the classical case and the appearance of magnetization plateaux in the quantum system ? Further; is the magnetic plateau accompanied by quantum order by disorder selection of a reduced manifold of states ? In this paper we have addressed these questions using a standard spin wave analysis, Lanczos diagonalization of finite clusters and a 2D Jordan-Wigner approach. We study the $X X Z \mathrm{AF}$ on the Kagomé lattice, where in general no soft modes are present. Our results show the persistence of the plateau at $1 / 3$ beyond the symmetric XXX point, and even the existence of a plateau for classical spins, in the easy axis case. This suggests that the existence of the soft modes is not a necessary condition for the existence of a plateau in the quantum case, although it does seem to extend the plateau region away from the Ising limit of $X X Z \mathrm{AF}$. The quantum ground state does indeed have a large projection onto a collinear state indicating a quantum order by disorder effect.

We define the $X X Z$ AF Hamiltonian

$$
H=J \sum_{<i, j>}\left(S_{i}^{x} S_{j}^{x}+S_{i}^{y} S_{j}^{y}+\Delta S_{i}^{z} S_{j}^{z}\right)-\vec{h} \cdot \sum_{i} \vec{S}_{i}
$$

where $\vec{S}_{i}=\left(S_{i}^{x}, S_{i}^{y}, S_{i}^{z}\right)$ is a vector of unit length, $J>0$ is the exchange constant and $0<\Delta<\infty$ the " $X X Z$ " anisotropy parameter. Specific values, $\Delta=1$ and $\Delta=0$ define the $X X X$ and $X X$ models respectively.

Considering first the $X X X$ case and defining $\vec{S}_{p}=$ $\sum_{i=1,2,3} \vec{S}_{i}$ as the total moment per triangular unit, the Hamiltonian for $N$ spins can be written as

$$
H=-N J S^{2}+\sum_{p=1}^{2 N / 3}\left(\frac{J}{2} \vec{S}_{p}^{2}-\frac{1}{2} \vec{h} \cdot \vec{S}_{p}\right) .
$$

Minimizing with respect to $\vec{S}_{p}$ gives $\vec{S}_{p}=\vec{h} /(2 J)$ and a net GS magnetization $\vec{M}=\vec{h} /(6 J S)$, for $|\vec{h}|<h_{c}=6 J S$. Above this field the spins are ferromagnetically aligned. 
In zero field this condition leads to a rigid coplanar spin configuration for each triangular plaquette, but the open nature of the Kagomé lattice allows freedom in the orientation of the triangular spin planes and a highly degenerate continuous GS manifold. Spin wave analysis shows that a branch of modes for fluctuations out of a discrete, but macroscopically large subset of collectively coplanar ground states become soft, leading to their thermal selection 14, 16, 23.

For non-zero magnetic field, a generic classical ground state for a single plaquette is not necessarily coplanar, in contrast to the zero field case 24. Selection of a collective coplanar state therefore requires selection at the level of each triangle, as well as between triangles.

This fact weakens, but does not destroy the coplanar selection [15]. For small field Zhitomirsky has proposed that a discrete set of coplanar states are selected with, on each triangle one spin, $A$, antiparallel to the field and spins $B$ and $C$ straddling the field direction in a " $Y$ " configuration. As in zero field, these states have soft modes associated with out of plane spin fluctuations and corresponding line defects in real space, allowing rotation of spins $B$ and $C$ about $A$.

As $h \rightarrow h_{c} / 3$ the two canted spins close up onto the field direction, forming collinear "up-up-down" (UUD) spin configurations on each triangle, with magnetization $M=1 / 3$. This special point has zero modes for in plane, as well as out of plane spin fluctuations [20,21]. The large number of soft modes leads to strong thermal selection of the UUD spin liquid 20,21.

To describe the fluctuations over the coplanar states near $h_{c} / 3$, we choose a coordinate system oriented with respect to local spin directions [14. At each site we choose right handed axes in spin space with the " $z$ " axis parallel to the spin and all " $x$ " axes mutually parallel, that is, perpendicular to the spin plane. The spin orientations are therefore parameterized by $\vec{S}_{i}=\left(\epsilon_{i}^{x}, \epsilon_{i}^{y}, 1-\alpha_{i}\right)$, with $\alpha_{i}$ determined from $\left|\vec{S}_{i}\right|=1$ and the quadratic part of the Hamiltonian reads

$$
1 / 2 \sum_{i, j}\left[M_{i, j}^{x} \epsilon_{i}^{x} \epsilon_{j}^{x}+M_{i, j}^{y} \epsilon_{i}^{y} \epsilon_{j}^{y}\right] .
$$

The only non-zero elements of the matrices $M^{x}$ and $M^{y}$ are the diagonal and nearest-neighbors terms. In particular, $M^{x}$ entries are $M_{i, i}^{x}=1$ and $M_{i, j}^{x}=1 / 2$ if $i$ and $j$ are nearest-neighbors. While $M^{x}$ turns out to be independent of $h$ and the particular GS we are considering, this is not the case for $M^{y}$.

The analysis of the " $x$ " sector is given in reference [14]. Even for disordered states the fundamental excitations are propagating modes, rather than the localized line defects that provide an alternative description of the soft fluctuations [23]. One can diagonalize (3) in Fourier space finding hidden branches of magnon-like excitations, as far as magnetic scattering techniques are concerned: an optical branch, an acoustic branch and a soft branch extending over the entire Brillouin zone. This branch is responsible for the selection of coplanar states in zero field and is present for all field values [15,21]. At the point $h / h_{c}=1 / 3$ and for fluctuations around an UUD state the " $y$ " sector also acquires a soft branch, independently of the GS. This fact plays in favor of a selection of the UUD states and leads to the UUD "spin-liquid" predicted and observed in reference [20].

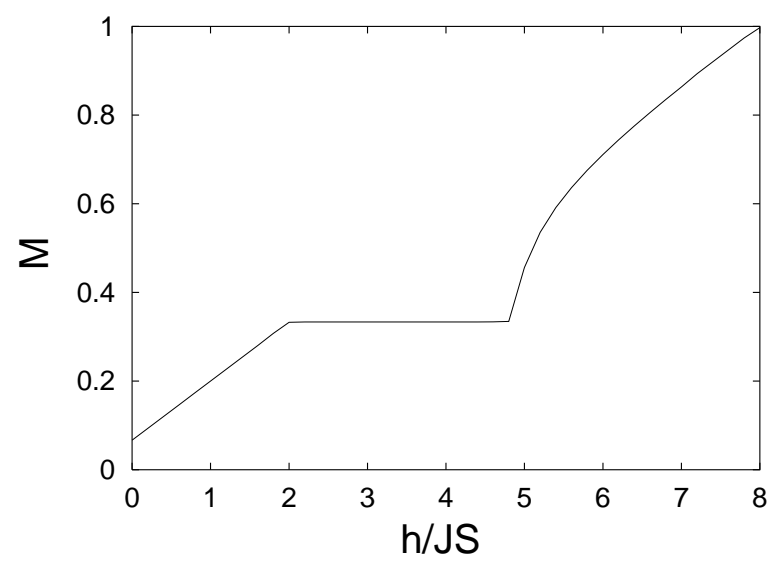

FIG. 1. Zero temperature magnetization curve, for classical spins. $\Delta=1.5$.

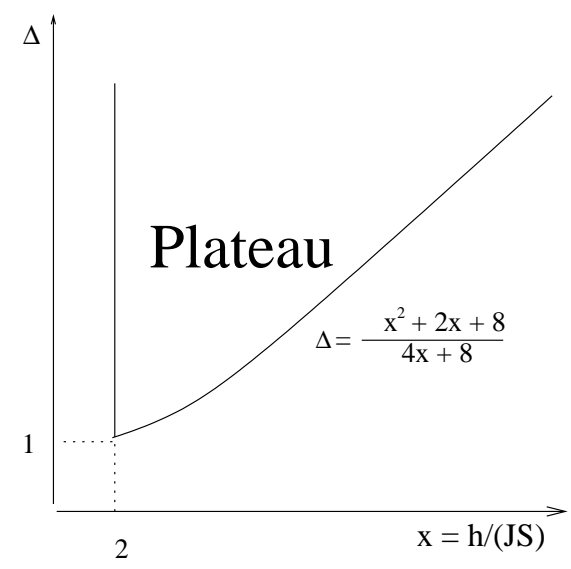

FIG. 2. Schematic classical phase diagram in the $h, \Delta$ plane.

The singular entropy of fluctuations driving the UUD spin liquid also leads to a magnetic anomaly and the beginnings of a magnetic plateau. Close to $h=h_{c} / 3$ the growing entropic contribution to the free energy pulls the minimum away from the ground state energy and towards the UUD point. This pulls the magnetization above the linear curve $M=h / h_{c}$ as one approaches $h_{c} / 3$ from below and pulls it below as one approaches from 
above. However the spin wave analysis gives no plateau, rather it gives a continuous relaxation away from linear behaviour and a point of inflection at $h=h_{c} / 3$. A remaining open question is whether nonlinear corrections can give a discontinuity and a finite plateau, as proposed in 21.

For $\Delta>1$ and in zero field the ground states remain highly degenerate and there are soft modes for out of plane fluctuations about collectively coplanar states with moment $M=(\Delta-1) / 3(\Delta+1)$ [25]. Adding a small field along the $z$ axis does not lift the degeneracy or remove the soft modes. The magnetization increases linearly until $M=1 / 3$ at $h=2 J S$, independently of $\Delta$. For higher fields, there is a plateau in the magnetization, at $M=1 / 3$, continuing out to $h=\left(2 \Delta-1+\sqrt{4 \Delta^{2}+4 \Delta-7}\right) J S$ where it starts to grow again, until reaching saturation at $h=(2+4 \Delta) J S$. The plateau occurs because, in this field region the field and exchange contributions to the Hamiltonian both vary quadratically with deformation away from an UUD configuration, leading to a linear instability. As a result the macroscopic, but discrete set of UUD states is selected energetically rather than entropically and the field must exceed $h=2 J S$ by a finite amount, before the ground state manifold leaves these configurations. The in-plane soft modes at $M=1 / 3$ are however a property of the $X X X$ point and are removed for $\Delta>1$. The lower boundary of the plateau tends to $M=0$ as one approaches the Ising limit, $\Delta \rightarrow \infty$, and there is a zero temperature plateau extending over the whole of the range $h<h_{c}$. The zero temperature magnetization for the classical case, is shown in Fig. 14 for $\Delta=1.5$. The data were obtained from zero temperature Monte Carlo, decreasing the field the starting value $h=h_{c}$. The plateau region is clearly visible. For $\Delta<1$ and zero field, the out of plane fluctuations acquire a finite stiffness [23]. In finite field the ground states are formed by canting the spins out of the plane perpendicular to the field direction. There are in general no soft modes and $M=1 / 3$ no longer has any special significance.

Classically, there is therefore an extended region in the $\Delta, h$ phase diagram at low temperature where plateau phenomena can be observed, extending from the point $\Delta=1, h=2 J S$ in a wedge, out to the line $\Delta=\infty, h<$ $h_{c}$, as is shown schematically in Fig. 2. A natural question to ask is therefore, do quantum fluctuations extend this region, leading to a plateau over a larger region of parameter space?

In trying to understand this problem, we present here a brief description of quantum fluctuations over coplanar states for arbitrary values of $M$. Within a HolsteinPrimakoff description for the spin variables, where the quantization axis is chosen according to the classical orientation of the spin described above, the $X X X$ Hamiltonian can be written:

$$
H=H_{0}+\frac{S}{2}\left(H_{2}+O(1 / \sqrt{S})\right)
$$

where $\mathrm{H}_{2}$ is a quadratic Hamiltonian of magnon creation and annihilation operators. The $O(1 / \sqrt{S})$ part contains higher order terms in these operators. By expressing the creation and annihilation operators in terms of the corresponding canonical variable $Q$ and $P, H_{2}$ reads [26]:

$$
H_{2}=1 / 2 \sum_{i, j}\left[M_{i, j}^{p} P_{i} P_{j}+M_{i, j}^{q} Q_{i} Q_{j}\right]
$$

where $M^{p}$ and $M^{q}$ are the matrices for classical fluctuations in the $y$ and $x$ sectors discussed above. There are therefore $N / 3 Q$ coordinates absent in $H_{2}$ with the corresponding canonical variables $P$ commuting with $\mathrm{H}_{2}$. This is the quantum analog of the flat branch. It can be explicitly shown to exist for the ordered coplanar GS with $q=0$ ordering vector, using a three color boson picture in momentum space. In this case, by an appropriate canonical transformation, $\mathrm{H}_{2}$ can be written as:

$$
H_{2}=\frac{1}{2} \sum_{\vec{k}, \mu}\left[P_{\mu}(\vec{k}) P_{\mu}(\overrightarrow{-k})+\omega_{\mu}(\vec{k}) X_{\mu}(\vec{k}) X_{\mu}(\overrightarrow{-k})\right]
$$

where $\mu$ is the color index and $\omega_{3}(\vec{k})=0 \forall \vec{k}$. We see then the presence of the flat mode in the quantum case, at this order in $1 / \sqrt{S}$, as a free particle like spectrum.

Of particular interest are the UUD states, where $N / 3$ $P$ coordinates are also absent from the Hamiltonian at this order. It is obvious that higher order terms of the Hamiltonian are crucial for understanding the spectrum of these excitations. In [27] it was shown how the zeropoint fluctuations of the fast variables (here $i=1,2$ ) introduce (via the higher order terms) an effective potential for the soft modes, apart from lifting the classical GS degeneracy. Following [27], processes like the tunneling effect between the, now discrete set of local minima can give rise to a finite, and even gaped stiffness of the soft modes. What we should point out in this example, is that the case $|M|=1 / 3$ corresponds to a different situation, where the effective dynamics of the soft modes introduced by the interactions with the normal oscillators will be radically different. We argue that, because of the absence of this huge number of canonical variables in $H_{2}$ for the UUD states, quantum fluctuations will indeed play in favor of a selection of these states, and to their stability as the magnetic field is changed away from $h_{c} / 3$. Such a selection, for a magnetic UUD state, is also present for the triangular lattice, due to zero-point fluctuations over an ordered classical ground state [19,28]. Following this idea one could follow a standard semiclassical expansion, and argue that if quantum fluctuations select a "Y" coplanar configuration for $M<1 / 3$ and a quasi-collinear configuration for $M>1 / 3$ as do thermal fluctuations in the classical case [21], the value of the field at which $M=1 / 3$ is reached from above and below will be renormalized at the quantum level. This scenario gives rise to a plateau at $M=1 / 3$ even for $\Delta=1$. Indeed, taking for example a $q=0$ structure, a straightforward numerical integration of the free bosonic branches involved 
either in the " $Y$ " or quasi-collinear Kagomé vacua, yields a finite plateau width. While this estimation is based on a particular ground-state $(q=0$ in this case), it is reasonable to assume a similar behavior for any coplanar ground-state. A more subtle point concerns the fact that this approach has to be taken with some care for the Kagomé model, because the oscillator frequencies have a non-analytic behavior at $h=2 J S$. This can give rise to divergencies in the coefficients of the formal power series and produce a non-analytic dependence on $S$ of the width of the plateau.

Motivated by these semiclassical arguments in favor of a plateau at $1 / 3$, we further analyze this issue using Lanczos diagonalization and a Jordan-Wigner approach, which are more appropriate in the deep quantum case 29. Let us first focus attention on the exact GS's obtained from the diagonalization of the clusters with periodic boundaries schematized in Fig. 3. Using a Lanczos algorithm [30] applied through all $S^{z}$ subspaces, we observe a plateau at $M=1 / 3$ as a function of magnetic field. Results for $N=18$ and all 24-spin clusters are shown in Fig. 4 for both $X X X$ and $X X Z$ with $\Delta>1$.

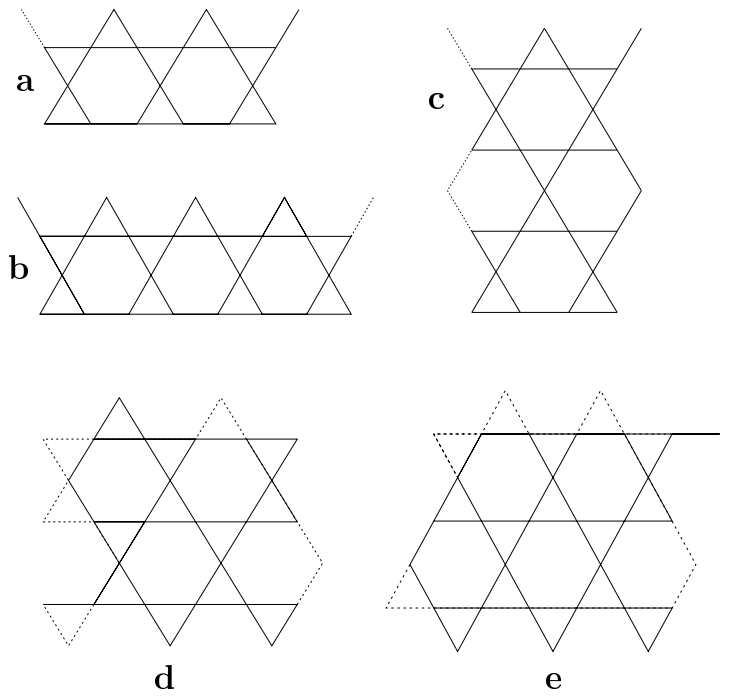

FIG. 3. Schematic view of clusters considered in exact diagonalizations. (a) 18 spins; (b), (c) and (d) 24 spins; (e) 27 spins. Dotted lines indicate PBC, holding throughout all boundaries.

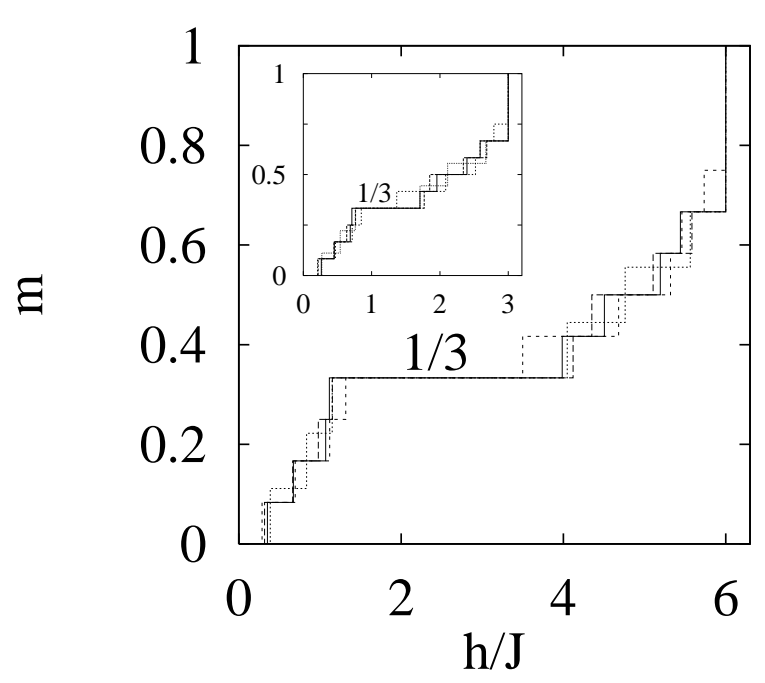

FIG. 4. Magnetization curves for $X X Z$ clusters $(\Delta=2.5)$. Dashed, solid, short dashed, and dotted lines denote respectively the results obtained for clusters (a), (b), (c) and (d). The inset displays the corresponding results for isotropic exchanges $(\Delta=1)$.

To check whether an UUD order actually occurs, we measured three-point coplanar correlation functions of the form $C_{\theta}(i, j, k)=\sum_{<i, j, k>}\left\langle\hat{n}_{i}^{\theta} \hat{n}_{j}^{-\theta}\left(1-\hat{n}_{k}^{z}\right)\right\rangle$ on the $M=1 / 3$ GS. Here, $\langle i, j, k\rangle$ denotes a cyclic permutation around an elementary triangle whereas, $\hat{n}^{z}=\sigma^{+} \sigma^{-}$ is the usual spin occupation number and $\hat{n}^{\theta}$ stands for the local density fields along the $\theta$-axes forming the coplanar " $Y$ " configuration. Specifically, after some elementary steps it can be readily shown that

$$
\begin{aligned}
C_{\theta}(i, j, k) & =\frac{1}{4} \sum_{<i, j, k>}\left\langle\left[-\sin ^{2} \theta \sigma_{i}^{x} \sigma_{j}^{x}+\left(1+\cos \theta \sigma_{i}^{z}\right)\right.\right. \\
& \left.\left.\times\left(1+\cos \theta \sigma_{j}^{z}\right)\right]\left(1-\hat{n}_{k}^{z}\right)\right\rangle .
\end{aligned}
$$

It turns out that $C_{\theta}$ increases monotonically with $\Delta$ and near the maximum depends very little either on the cluster size, its particular shape, or the plaquette location. A large but unsaturated value of this parameter at $\theta=0$, as is shown in Fig. 5, is consistent with the idea of a collinear UUD spin liquid allowing a finite probability 
for configurations with defects.

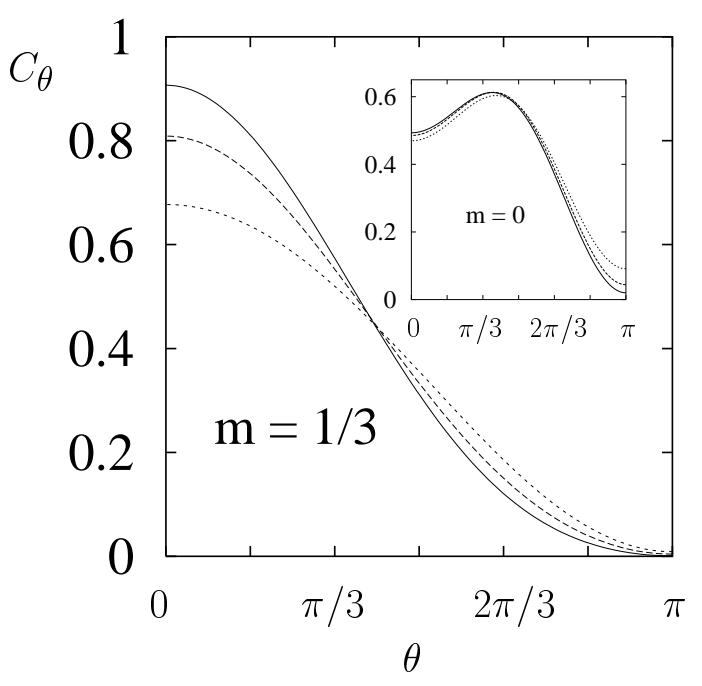

FIG. 5. Triangular correlation functions measuring variations around the coplanar "Y" configurations at $M=1 / 3$ in cluster (d). Solid, dashed and dotted lines refers to $\Delta=2.5,1$, and 0 respectively. For comparison, the inset shows the corresponding situation a $M=0$, favoring a classical behavior.

One can follow this idea further by testing if the system selects a single state or microscopic number of states making up the UUD spin liquid. By sorting the probabilities of the ground state components in real space, we find that the latter exhibit a sharply peaked distribution, at the top of which there are a number of UUD states. This is shown schematically in Fig. 6 for cluster (e). The weight distribution becomes even sharper for $\Delta>1$ and broadens steadily upon decreasing the anisotropy. Similar results were observed in the remaining clusters displayed in Fig. 3 [31]. This ensemble of results therefore gives a strong indication that there is quantum order by disorder selection of the UUD spin liquid over the plateau region for $\Delta=1$. The narrowing for $\Delta>1$ is evidence for the removal of non-collinear fluctuations away from the symmetric $X X X$ point, as one might expect from the energetic arguments presented earlier.

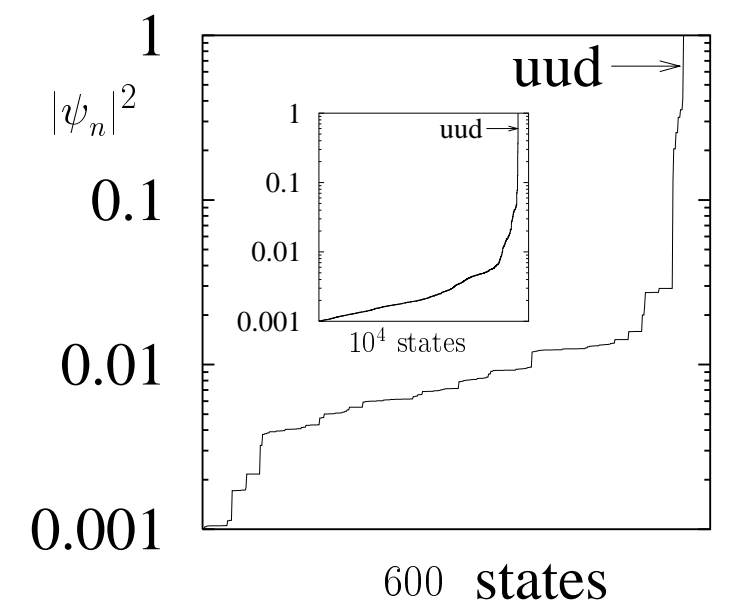

FIG. 6. Ground state of cluster (e) for $M=1 / 3$ and $\Delta=2.5$. Real space states are sorted by increasing weights and normalized to their maximum value (up-up-down configurations). The inset exhibits the ground state components for $\Delta=1$. Only weights above $10^{-3}$ are shown.

As a final step, we now study our system by means of a generalization of the well known Jordan-Wigner transformation in $1 \mathrm{D}$, which was originally proposed in 32 and further developed in 33 35. It consists of a mapping of the original spin variables into spinless fermion variables $c_{i}$ via $S_{i}^{-}=c_{i} \exp i \sum_{j \neq i} c_{j}^{\dagger} c_{j} \arg \left(\tau_{j}-\tau_{i}\right)$, where $\tau_{i}=$ $x_{i}+i y_{i}$ is the complex coordinate and $S_{i}^{z}=c_{i}^{\dagger} c_{i}-1 / 2$. The advantage of this transformation is that it imposes exactly the single occupancy constraint, but at the price of introducing a minimal coupling with Chern-Simons gauge fields. One usually proceeds by a mean field decoupling to end up with a problem of fermions on the given lattice in a properly chosen magnetic background, which can be solved using standard techniques. In this language, an external magnetic field applied to the spin system enters as a chemical potential for the spinless fermions. In the present case and mainly motivated by the tendency of the AF Kagomé lattice to disorder, we have chosen a homogeneous mean field, that is a uniform fermion density $c_{j}^{\dagger} c_{j} \equiv \frac{1}{2}(1+M), \forall j$. We restricted the analysis to the case $\Delta=0$ where this calculation scheme becomes most reliable. The resulting magnetization curve is shown in Fig. 7. A clear plateau again shows up at $M=1 / 3$. Two other plateaux also appeared at $M=0$ and $M=2 / 3$ which could be an artifact of the mean field approximation and more work is needed to be conclusive at this point. 


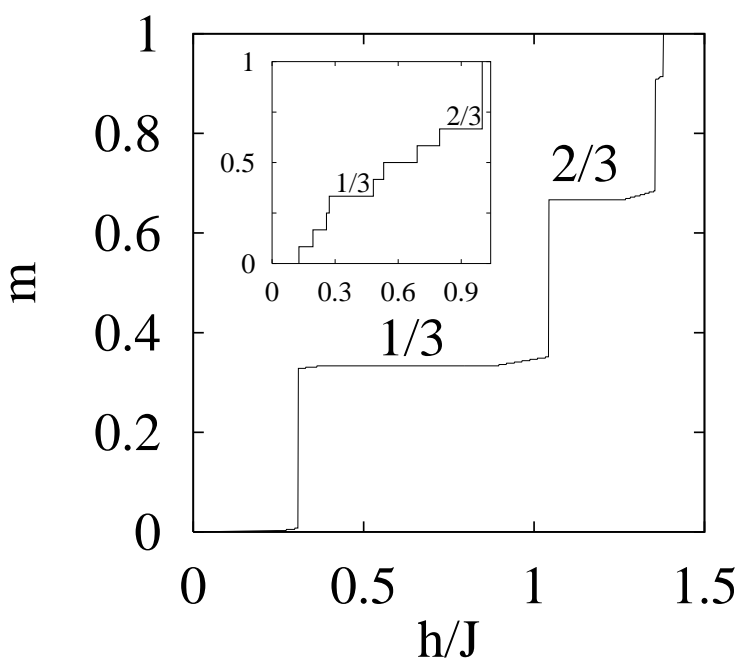

FIG. 7. Mean field magnetization curves for $X X$ lattices, using homogeneous fermion densities. For comparison, the inset shows the exact magnetization curve of cluster (c) using $\Delta=0$.

It is interesting to recall that the $1 / 3$ plateau is expected to disappear for low enough $X X Z$ anisotropy in the triangular lattice [5], while our mean-field results suggest that it persists even in the $X X$ limit of the Kagomé lattice. This result is again consistent with the idea that quantum fluctuations stabilize a plateau over an extensive range of parameter space. The Lanczos magnetization curve at $\Delta=0$ yields no conclusive evidence on this issue, though there is evidence that the sharp weight structure of the GS components disappears.

To summarize, we have studied the magnetization behavior of both classical and quantum Kagomé AF using a variety of methods. In all cases our results suggest that at least for $\Delta \geq 1$, there is selection of an UUD state which is ultimately responsible for the emergence of a magnetization plateau at $M=1 / 3$. An additional feature, particular to the Kagomé lattice is that the UUD state is not a Néel ordered state, rather it is a spin liquid. Our evidence for this is that we find a number of states with comparable weights. It seems therefore that the strong fluctuations associated with the the spin liquid state do not destroy the plateau; rather, the two effects co-habit. Further investigation of these issues is underway. At the classical level a plateau occurs in a region of $(\Delta, h)$ phase space, terminating at the point $(\Delta=1, h=2 J S)$, where a pseudo plateau is driven by the entropic contribution to the free energy. The results of this paper allow us to speculate that quantum fluctuations stabilize a plateau over a more extensive region of parameter space. The robustness of this result appears to be associated with a clean separation of the GS components according to their weight scale, the highest of which are UUD configurations.

We aknowledge useful discussions with J.T. Chalker, D. Champion, P. Degiovanni, F. Delduc, E. Fradkin, L. Freidel, A. Honecker, O. Petrenko, G.L. Rossini and M. Zhitomirsky and the financial support of the ECOS-Sud committee. The research of D.C.C and M.D.G. is partially supported by CONICET and Fundación Antorchas, Argentina (grant No. A-13622/1-106).

[1] For reviews consult, E. Dagotto, T.M. Rice, Science 271, 618 (1996); T.M. Rice, Z. Phys. B103, 165 (1997).

[2] M. Oshikawa, M. Yamanaka, I. Affleck, Phys. Rev. Lett. 78, 1984 (1997).

[3] D.C. Cabra, A. Honecker, P. Pujol, Phys. Rev. Lett. 79, 5126 (1997); Phys. Rev. B 58, 6241 (1998).

[4] K. Totsuka, Phys. Rev. B 57, 3454 (1998).

[5] A. Honecker, J. Phys.: Condens. Matter 11, 1697 (1999).

[6] K. Onizuka, H. Kageyama, Y. Narumi, K. Kindo, Y. Ueda, T. Goto, J. Phys. Soc. Jpn. 69, 1016 (2000).

[7] T. Momoi, K. Totsuka, Phys. Rev. B 61, 3231 (2000); Phys. Rev. B 62, 15067 (2000).

[8] E. Müller-Hartman, R.R.P. Singh, C. Knetter, G.S. Uhrig, Phys. Rev. Lett. 84, 1808 (2000).

[9] G. Misguich, Th. Jollicoeur, S.M. Girvin, Phys. Rev. Lett. 87, 097203 (2001).

[10] R. Moessner, preprint cond-mat/0010301.

[11] J. Villain, R. Bidaux, J.P. Carton, R.J. Conte, J. Phys. (Paris), 41, 1263, (1980).

[12] E.F. Shender, Sov. Phys. JETP 56, 178, (1982).

[13] H. Shultz, T. Ziman in "Magnetic systems with competing interactions" Ed. H.T. Diep, (World Scientific, Singapore, 1994).

[14] J.T. Chalker, P.C.W. Holdsworth, E.F. Shender, Phys. Rev. Lett. 68, 855 (1992)

[15] E.F. Shender, P.C.W. Holdsworth In Fluctuations and Order: a new synthisis, M.M. Millonas ed. SpringerVerlag, 1996

[16] R.J.N. Reimers, A.J. Berlinsky, Phys. Rev. B48, 9539, (1993).

[17] See e.g. H. Asakawa, M. Suzuki, Int. J. Mod. Phys. B 9, 933 (1995) and references therein.

[18] C. Waldtmann H. -U. Everts, B. Bernu, P. Sindzingre, C. Lhuillier, P. Lecheminant, L. Pierre, Eur. Phys. J. B 
2, 501 (1998).

[19] D.I. Golosov, A.V. Chubukov, Pis'ma ZhETF 50, 416 (1989) [JETP Lett. 50, 451 (1989)], A. V. Chubukov, D. I. Golosov, J. Phys.: Condens. Matter 3, 69 (1991).

[20] M. E. Zhitomirsky, A. Honecker, O. A. Petrenko, Phys. Rev. Lett. 85, 3269 (2000).

[21] M.E. Zhitomirsky, preprint cond-mat/0107608.

[22] K. Hida, preprint cond-mat/0106498.

[23] I. Ritchey, P. Chandra, P. Coleman, Phys. Rev. B 47, 15342 (1993).

[24] As in the case of a tetrahedral plaquette, see: R. Moessner, J.T. Chalker, Phys. Rev. Lett. 80, 2929, (1998).

[25] S.T. Bramwell, M.J.P. Gingras, J.N. Reimers, unpublished.

[26] A.B. Harris, C. Kallin, A.J. Berlinsky, Phys. Rev. B 45, 2899 (1992).

[27] B. Douçot, P. Simon, J. Phys. A 31, 5855 (1998).

[28] H. Kawamura, S. Miyashita J. Phys. Soc. Jpn. 54, 4530 (1985).

[29] See for instance, P. Lecheminant, B. Bernu, C. Lhuillier, L. Pierre, Phys. Rev. B 52, 6647 (1995).

[30] See, for example G.H. Golub, C.F. Van Loan, "Matrix Computations", 3rd edition (Johns Hopkins University Press, Baltimore 1996).

[31] Note that periodic boundaries are essential; free boundary effects yield neither evidence of massive spin excitations nor of sharp weight distributions.

[32] E. Fradkin, Phys. Rev. Lett. 63, 332 (1989); J. Ambjorn, G. Semenoff, Phys. Lett. B226, 107 (1989).

[33] Y.R. Wang, Phys. Rev. B 43, 3786 (1991); Phys. Rev. B 45, 12604 (1992); Phys. Rev. B 45, 12608 (1992).

[34] K. Yang, L.K. Warman, S.M. Girvin, Phys. Rev. Lett. 70, 2641 (1993).

[35] A. López, A.G. Rojo, E. Fradkin, Phys. Rev. B 49, 15139 (1994). 